02

\title{
Влияние радиационных дефектов различного типа на критический ток слоистого анизотропного сверхпроводника
}

\author{
() А.Н. Максимова, В.А. Кашурников, А.Н. Мороз, И.А. Руднев \\ Национальный исследовательский ядерный университет „МИФИ“, \\ Москва, Россия \\ E-mail: anmaksimova@mephi.ru
}

Поступила в Редакцию 16 июля 2021 г.

В окончательной редакции 16 июля 2021 г.

Принята к публикации 18 июля 2021 г.

\begin{abstract}
Численными методами исследовано влияние радиационных дефектов на критический ток слоистого анизотропного сверхпроводника. Рассмотрены модельные дефекты, имитирующих различные случаи радиационных повреждений сверхпроводника в результате электронного и ионного облучений. Полученные зависимости критического тока от магнитного поля показывают, что наибольшее усиление пиннинга при равном флюенсе облучения дают дефекты, образующие объемные конические области внутри образца. Показано, что в слабоанизотропном сверхпроводнике конические дефекты эффективнее повышают критический ток, чем в сильноанизотропном. Рассмотрено взаимодействие вихревой решетки с радиационными дефектами, получены вихревые конфигурации, возникающие под действием собственного поля транспортного тока.
\end{abstract}

Ключевые слова: ВТСП, пиннинг, вихри Абрикосова, радиационные дефекты, метод Монте-Карло.

DOI: 10.21883 /FTT.2021.11.51584.169

\section{1. Введение}

Несмотря на значительное количество исследований, повышение пиннинга в высокотемпературном сверхпроводнике (ВТСП) остается важной задачей. Современные ВТСП-ленты второго поколения, например, на основе соединений редких земель $(R \mathrm{eBaCuO}$, где $R e-$ редкая земля), обладают высокими значениями критического тока, благодаря чему они широко используются в электроэнергетике, позволяя создавать высокоэффективные линии электропередачи, моторы и генераторы. ВТСП-ленты также обладают способностью сохранять высокий критический ток в магнитных полях, что дает возможность использовать их в ускорителях и токамаках, где они подвергаются воздействию излучения. Как показывают экспериментальные исследования, под действием излучения в сверхпроводнике образуются дефекты различного типа, которые могут являться центрами пиннинга для вихрей. Таким образом, излучение влияет на магнитные и транспортные свойства сверхпроводника, и это также означает возможность изменять свойства образца, целенаправленно облучая его частицами различного типа. На практике используется облучение нейтронами [1], протонами [2], электронами [3], $\gamma$-квантами [4] и тяжелыми ионами [5]. При этом форма дефекта зависит от энергии и типа облучающих частиц. При облучении электронами и $\gamma$-квантами возникают преимущественно точечные центры пиннинга, при облучении тяжелыми ионами дефект может быть как в форме прямолинейного трека радиусом порядка длины когерентности (энергия иона в интервале от сотен Mev до нескольких $\mathrm{Gev}$ ), так и областью значительного размера (каскад), не пронизывающей пленку насквозь. Такой дефект возникает, если энергия облучающего иона меньше или порядка нескольких Mev на нуклон [6], а толщина ВТСП слоя больше проективного пробега ионов. Характер дефектообразования в ВТСП можно оценить с помощью широко используемого пакета SRIM (Stopping and Range of Ions in Matter) [7]. Так, например, в [8] представлены результаты расчета концентрации радиационных дефектов в ВТСП для некоторых типов ионов. Приближенно в численном расчете каскад дефектов может быть модельно рассмотрен как конус с вершиной на поверхности пленки. Глубина такого дефекта зависит от энергии облучающего иона и, следовательно, может регулироваться.

Как показывают многочисленные исследования, облучение при малых флюенсах может приводить как повышению, так и понижению критического тока. На практике, ситуация зависит от величины исходного критического тока сверхпроводника, а также типа и флюенса облучения. Повышение критического тока при увеличении концентрации дефектов (рис. 1) связано с образованием новых центров пиннинга, понижение обусловлено рядом факторов, которые будут отмечены ниже. Хотя в настоящей статье нас интересует первая область повышение критического тока, мы кратко дадим обзор экспериментальных данных, касающихся как первой, так и второй области.

В работах [9-15] исследована деградация сверхпроводящих свойств ВТСП-ленты при облучении нейтронами. Установлено, что быстрее ленты деградируют при высоких рабочих температурах и в магнитном поле, параллельном плоскости ленты. Также показано, 


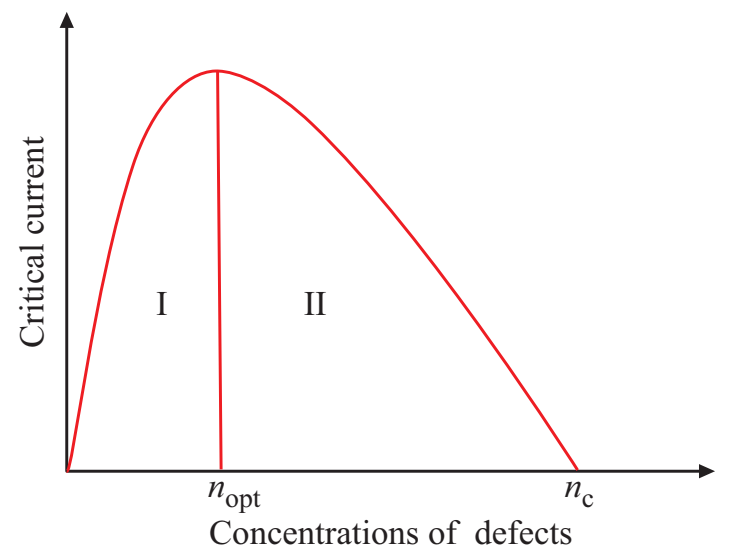

Рис. 1. Качественная зависимость критического тока от концентрации дефектов для сверхпроводников второго рода. I - область повышения критического тока, II - область падения критического тока.

что при низком флюенсе облучения наблюдается повышение критического тока, которое при определенном значении $\left(1.2 \cdot 10^{22} \mathrm{~m}^{-2}\right)$ достигает максимума. Влияние нейтронного облучения на ВТСП-ленты, содержащие искусственные центры пиннинга, было проведено в работе [14]. Показано, что падение критической температуры определяется только величиной флюенса и не зависит от наличия искусственных дефектов, в то время как критический ток при наличии искусственных дефектов деградирует при более низком флюенсе. Показано также, что при высоких флюенсах $\left(2.3 \cdot 10^{22} \mathrm{~m}^{-2}\right)$ объемный пиннинг вихрей определяется только радиационными дефектами.

Если при облучении для малых флюенсов наблюдается рост критического тока, то по достижении определенного, зависящего от конкретного сверхпроводника, флюенса облучения (например, для облучения $\mathrm{YBa}_{2} \mathrm{Cu}_{3} \mathrm{O}_{7+\delta}$ (YВСО) быстрыми нейтронами это значение составляет $\sim 10^{22} \mathrm{~m}^{-2}$ [14], см. также [15]) рост криттока сменяется его деградацией. Частично это связано со снижением критической температуры при росте концентрации дефектов, частично - с перекрытием их потенциальных ям [16]. Той же самой зависимости от облучения подчиняется и поле необратимости [14]. Еще одним эффектом является подстройка решетки вихрей под решетку дефектов ([17], где наблюдались минимумы на зависимости магнитосопротивления от внешнего поля при полях подстройки). В [18] исследовалось влияние облучения на скорость релаксации в сверхпроводнике $\mathrm{CaFe}_{2} \mathrm{As}_{2}$. Влияние облучения на величину критического тока исследовалось с начала 1990-х [19-21], но продолжает быть актуальным и в настоящее время [22-24]. В [19] измерялись зависимости критического тока от магнитного поля, а также поля необратимости. Показано повышение (при всех температурах) поля необратимости (параллельного оси анизотропии) при облучении. Однако в плоскости сверхпроводящих слоев поле необратимости, наоборот, при облучении снижается. Наблюдалось подавление эффекта рыбьего хвоста для плотности критического тока. В [20] исследовалось влияние на критический ток $\mathrm{YBa}_{2} \mathrm{Cu}_{3} \mathrm{O}_{7+\delta}$ облучения быстрыми нейтронами. В работе [21] для расчета критического тока в магнитном поле использовался метод молекулярной динамики, комбинированный с теорией Гинзбурга-Ландау. Для ҮВСО с колончатыми радиационными дефектами получены убывающие зависимости критического тока от магнитного поля. Исследовано влияние регулярного и случайного распределения дефектов.

Облучение с достаточно высоким флюенсом снижает критическую температуру сверхпроводящего перехода. $\mathrm{B}$ работе [22] образец $\mathrm{YBa}_{2} \mathrm{Cu}_{3} \mathrm{O}_{8+\delta}$ облучался протонами, таким образом, что получившиеся дефекты формируют регулярную решетку. Получены локальные пики критического тока от магнитного поля, соответствующие полям подстройки. Получены распределения локальной критической температуры. В [23] зависимости криттока от магнитного поля были получены для $\mathrm{MgB}_{2}$, в [24] — для $\mathrm{La}_{1.5} \mathrm{Dy}_{0.5} \mathrm{CaBa}_{2} \mathrm{Cu}_{5} \mathrm{O}_{\mathrm{z}}(\mathrm{La}-2125)$.

Исследование воздействия облучения тяжелыми ионами инертных газов $[25,26]$ показывают незначительный рост критического тока при низких флюенсах с последующим монотонным снижением вплоть до полного исчезновения сверхпроводящих свойств уже при флюенсе $\sim 10^{17} \mathrm{~m}^{-2}$. Как показывают исследования, радиационная стойкость ВТСП-лент по отношению к облучению ${ }^{132} \mathrm{Xe}^{27+}(167 \mathrm{MeV}),{ }^{132} \mathrm{Xe}^{27+}(80 \mathrm{MeV})$ и ${ }^{84} \mathrm{Kr}^{17+}$ $(107 \mathrm{MeV})$ составляет $1 \cdot 10^{12} \mathrm{~cm}^{-2}, 35 \cdot 10^{12} \mathrm{~cm}^{-2}$ и $1 \cdot 10^{13} \mathrm{~cm}^{2}$ соответственно. Также были проведены исследования с использованием ионов различных элементов: кислород [27-29], цирконий [30], свинец [31]. Было показано, что при определенных условиях воздействие пучком ионов кислорода в полях порядка 5 Т возможно добиться повышения критического тока в 2 раза.

В отличие от эксперимента, численное моделирование позволяет детально проанализировать механизм пиннинга на уровне отдельных вихрей и разделить вклад различных факторов в формирование критического тока. В работе [21] и во многих других был выполнен подробный численный анализ влияния концентрации и расположения дефектов на величину критического тока в магнитном поле. Однако сделано это было для колончатых дефектов, соответствующих облучению ионами высокой энергии. Численные исследования для радиационных дефектов другой формы в литературе практически отсутствуют.

Цель нашей работы заключается в моделировании влияния на эффективность пиннинга и критический ток ВТСП некоторых типов трехмерных радиационных дефектов, возникающих при различной энергии облучения. Мы рассмотрим дефекты в виде аморфизированного трека (колончатый тип дефектов), точечные дефекты и каскадные дефекты. Особенностью нашего рассмотрения является прямой учет трехмерной структуры, который, 
как мы покажем, дает возможность моделирования пиннинга на трехмерных структурных дефектах, а именно прямолинейных треках, каскадах и точечных дефектах. Статья построена следующим образом. В разделе 2 описана модель слоистого ВТСП и поясняется способ введения описания взаимодействия вихря с радиационным дефектом в алгоритм. В 3 приводятся результаты расчета зависимости критического тока от магнитного поля и их обсуждение. В заключении сформулированы основные результаты работы.

\section{2. Модель и метод расчета}

Расчеты выполнены методом Монте-Карло для трехмерной модели слоистого ВТСП [32-35]. В рамках данной модели вихревая нить рассматривается как стопка плоских взаимодействующих слоевых вихрей - панкейков. Энергия системы вихрей имеет вид

$$
\begin{aligned}
G= & \sum\left\{N_{z} \varepsilon+\sum_{i<j} U_{\text {in-plane }}\left(r_{i j}\right)+\sum_{i, j} U_{p}\left(r_{i j}\right)\right. \\
& \left.+\sum_{i, j} U_{\text {surf }}\left(r_{i j}^{i m}\right)+\sum_{i} U_{\text {inter-plane }}\left(r_{i}^{z, z+1}\right)\right\},
\end{aligned}
$$

где $\left.\varepsilon=d \varepsilon_{0}(\ln [\lambda(T) / \xi T)]+0.52\right)-$ собственная энергия вихря, $\lambda(0), \xi(0)$ - глубина проникновения и длина когерентности при $T=0 ; N_{z}$ - число панкейков в плоскости $z$; второй член описывает попарное взаимодействие панкейков, третий - взаимодействие вихрей с центрами пиннинга, четвертый - взаимодействие вихрей с поверхностью и мейсснеровским и транспортным током, последний - межплоскостное взаимодействие панкейков; $\varepsilon_{0}=\Phi_{0}^{2} /(4 \pi \lambda)^{2}, \Phi_{0}=\pi \hbar c / e-$ квант магнитного потока. Для межплоскостного взаимодействия нами была использована форма потенциала, полученная в работах $[36,37]$ :

$$
U_{\text {inter-plane }}\left(r_{i}^{z, z+1}\right)=U_{\text {em }}\left(r_{i}^{z, z+1}\right)+U_{j o s}\left(r_{i}^{z, z+1}\right),
$$

$U_{e m}$ - электромагнитное, $U_{j o s}-$ джозефсоновское взаимодействие панкейков, расположенных в соседних слоях. Данные слагаемые имеют вид

$$
U_{e m}\left(r_{i}^{z, z+1}\right)=2 d \varepsilon_{0}\left\lfloor C+\ln \left(r_{i}^{z, z+1} / 2 \lambda\right)+K_{0}\left(r_{i}^{z, z+1} / \lambda\right)\right\rfloor,
$$

$\mathrm{C}=0.5772-$ постоянная Эйлера.

$$
U_{j o s}^{z, z+1}\left(r_{i}^{z, z+1}\right)=\left\{\begin{aligned}
\varepsilon_{0} d[1 & +\ln (\lambda / d)] 0.25\left(r_{i}^{z, z+1} / r_{g}\right)^{2} \\
& \times \ln \left(9 r_{g} / r_{i}^{z, z+1}\right), \quad r_{i}^{z, z+1} \leq 2 r_{g}, \\
\varepsilon_{0} d[1+\ln (\lambda / d)]\left[\left(r_{i}^{z, z+1} / r_{g}\right)-0.5\right], & r_{i}^{z, z+1}>2 r_{f},
\end{aligned}\right.
$$

$r_{g}=\gamma d-$ характерное расстояние джозефсоновского взаимодействия, $\gamma$ - параметр анизотропии, $d-$ межплоскостное расстояние.
Вследствие ограничений по быстродействию и оперативной памяти компьютера в расчете Монте-Карло удается рассмотреть стопку из не более чем 20 плоскостей. В то же время минимальная толщина ВТСП-пленки, используемой на практике, - порядка нескольких сотен $\mathrm{nm}$. В качестве выхода можно объединить несколько панкейков в соседних плоскостях и применять подпроцессы рождения, уничтожения и движения к стопке панкейков как к единому объекту. Приближенно такой подход возможен, т.к. реальные масштабы изгибов абрикосовских вихрей определяются параметрами упругости вихревой нити [38] и обычно много больше межслоевого расстояния. В нашей работе за единый объект принята стопка из 10 слоевых вихрей, таким образом, рассматривается система из 200 ВТСП-слоев.

Любой сверхпроводник всегда имеет дефекты структуры, которые работают как центры пиннинга для вихрей. Такой дефект называется собственным и вводится в расчет как малая область (размером порядка длины когерентности) с подавленными сверхпроводящими свойствами. Дополнительно к собственным центрам пиннинга образец может содержать искусственные радиационные дефекты. В нашей работе мы исследуем влияние на вихревую решетку дефектов, возникающих при облучении сверхпроводника электронами и ионами различной энергии, для определенности, ионами ксенона [39]. Конфигурации дефектов, возникающих при этом, показаны схематично на рис. 2. Если энергия ионов $\sim 1 \mathrm{Gev}$,

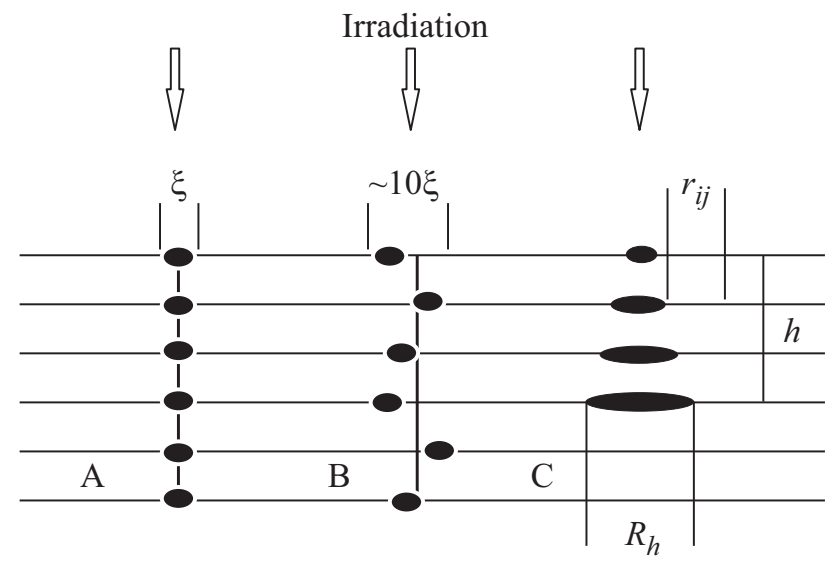

Рис. 2. Схемы радиационных дефектов при облучении заряженными частицами различной энергии и электронами. Горизонтальными линиями показаны ВТСП-слои. Направление оси дефекта совпадает с направлением пучка частиц излучения. $a$ - Колончатый дефект (на дальнейших рисунках col-str), возникает при облучении ионами с энергией от нескольких сотен Mev до $1 \mathrm{Gev.} b-$ Совокупность точечных дефектов, расположенных случайным образом в пределах некоторого расстояния от оси (на дальнейших рисунках col-rand). Возникает при облучении электронами. $c-$ Конический дефект. Возникает при облучении ионами с энергией от нескольких Mev до нескольких десятков Mev. Внутрь конического дефекта вихрь проникнуть не может, но может закрепляться на границе. 
дефект имеет вид прямолинейного трека диаметром $\sim \xi$, пронизывающего пленку насквозь (рис. $2, a$ ). Если энергия иона $\sim$ нескольких Mev, дефект не является сквозным и может быть описан как расширяющийся вглубь конус, глубина и радиус основания которого зависят от энергии иона (рис. 2,c). Радиус в нижней части может достигать $\lambda$. Примем, что такой дефект является областью, внутрь которой вихрь не может проникнуть, однако может закрепляться на границе. Центр пиннинга, возникающий при облучении электронами, может быть описан как совокупность точечных дефектов, расположенных в каждом слое и отстоящих от оси на случайное расстояние. Примем для определенности, что это расстояние не превышает $10 \xi$ (рис. $2, b$ ). Дефекты, соответствующие рис. $2, b$, рассматривались в работе [22]. Сверхпроводник также имеет собственные центры пиннинга, расположенные случайным образом и имеющие радиус $\sim \xi$. Потенциал взаимодействия вихря с дефектом моделируется как

$$
U_{p m}=-\alpha \frac{1}{1+r_{i j} / \xi} \exp \left(\frac{r_{i j}}{2 \xi}\right)
$$

где $r_{i j}$ - расстояние до центра дефекта или до края дефектной области для конического дефекта (рис. 2,c), $\alpha$ - эффективная глубина потенциальной ямы дефекта. Данная форма потенциальной ямы принята одинаковой для собственного и радиационного дефекта. Глубина ямы радиационного дефекта принята на порядок большей глубины ямы собственного дефекта (слабый собственный пиннинг).

\section{3. Результаты и обсуждение}

Для моделирования нами были выбраны параметры ВТСП на основе висмута $\mathrm{Bi}_{2} \mathrm{Sr}_{2} \mathrm{CaCu}_{2} \mathrm{O}_{8-\delta}$ (BSCCO, $\left.\gamma(0)=180 \mathrm{~nm}, \xi(0)=2 \mathrm{~nm}, T_{c}=84 \mathrm{~K}\right)$. Поскольку целью работы является анализ взаимодействия вихревой решетки с радиационными дефектами, то для исключения влияния тепловых флуктуаций примем $T=1 \mathrm{~K}$. Размер образца в плоскости $a b$ составляет $5 \times 3 \mu \mathrm{m}$, толщина пленки $c=540 \mathrm{~nm}$. При этом в образце находится в среднем порядка 1000 вихревых нитей. Как показывают наши предыдущие расчеты, результаты, полученные для малой системы, могут быть качественно экстраполированы на реальные экспериментальные размеры. Образец содержит $N_{h}=20$ радиационных дефектов, образующих треугольную решетку. Конические радиационные дефекты имеют радиус в самой широкой части $R_{h}=200 \mathrm{~nm}$ (это больше глубины проникновения магнитного поля в сверхпроводник). Радиус конического дефекта на поверхности пленки - порядка $\xi$. Это не ограничит общность выводов, но позволит свести к минимуму погрешность, связанную с различным возможным расположением дефектов. Концентрация радиационных дефектов принята равной $1.33 \cdot 10^{12} \mathrm{~m}^{-2}$ ),

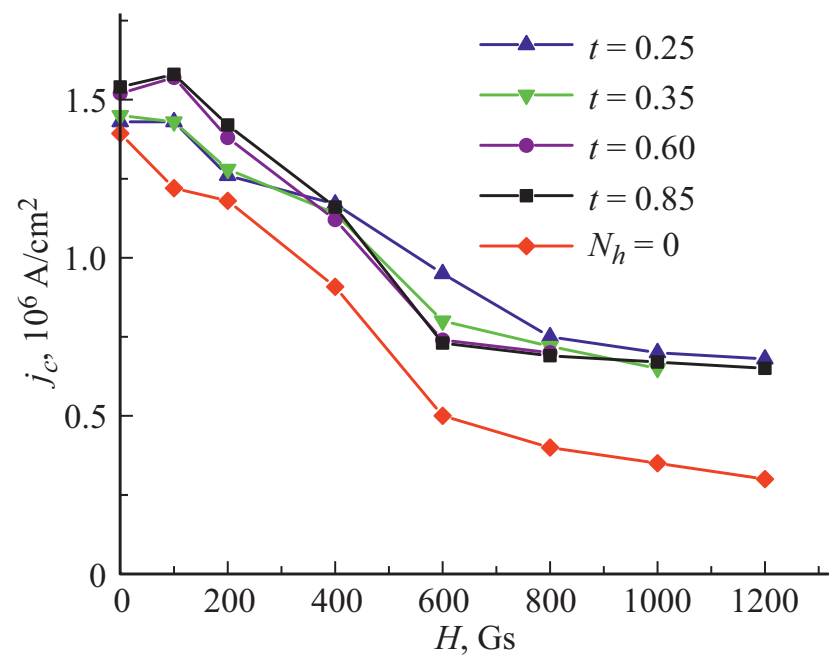

Рис. 3. Зависимость критического тока от глубины дефекта. Параметр $t=h / c$ характеризует относительную глубину конического дефекта. Абсолютная погрешность критического тока в данном расчете имеет порядок $0.01 \mathrm{~A} / \mathrm{cm}^{2}$ (оценена по трем расчетам, отличающимся расположением собственных дефектов).

что по порядку величины соответствует радиационной стойкости ВТСП при облучении ксеноном.

На рис. 3 показаны расчетные зависимости критического тока от магнитного поля при различной глубине $h$ конических дефектов, зависящей от энергии облучающих ионов. ВТСП на основе висмута являются сильноанизотропными веществами, поэтому на рис. 3-4 расчеты сделаны при параметре анизотропии $\gamma=250$.

Видно, что при всех значениях магнитного поля критический ток повышается примерно на $30 \%$. При этом глубокие дефекты более эффективны при низких полях.

Сравним эффективность конических дефектов с эффективностью колончатых (col-str) и колончатых с разбросом отдельных точечных вокруг его оси (col-rand, рис. 4). В данном расчете количество колончатых прямых и колончатых с разбросом дефектов совпадает с количеством конических и равно 20. В этом случае расчет показывает, что как при сильном, так и при слабом магнитном поле повышение критического тока для колончатых и случайных дефектов в среднем порядка $10 \%$, т.е. при равном флюенсе конические дефекты более эффективны.

При переходе к сверхпроводнику с низкой анизотропией (YBCO, $\gamma=10$ ) замена характерных длин не приведет к качественным изменениям. Поэтому просто заменим в нашем расчете $\gamma=250$ на $\gamma=10$. Результаты при промежуточной глубине дефектов показаны на рис. 5. При выбранной конфигурации собственных дефектов в полях порядка 1000 Gs удается добиться повышения критического тока в 1.5 раза, при этом в нулевом поле повышение составляет примерно $40 \%$. 


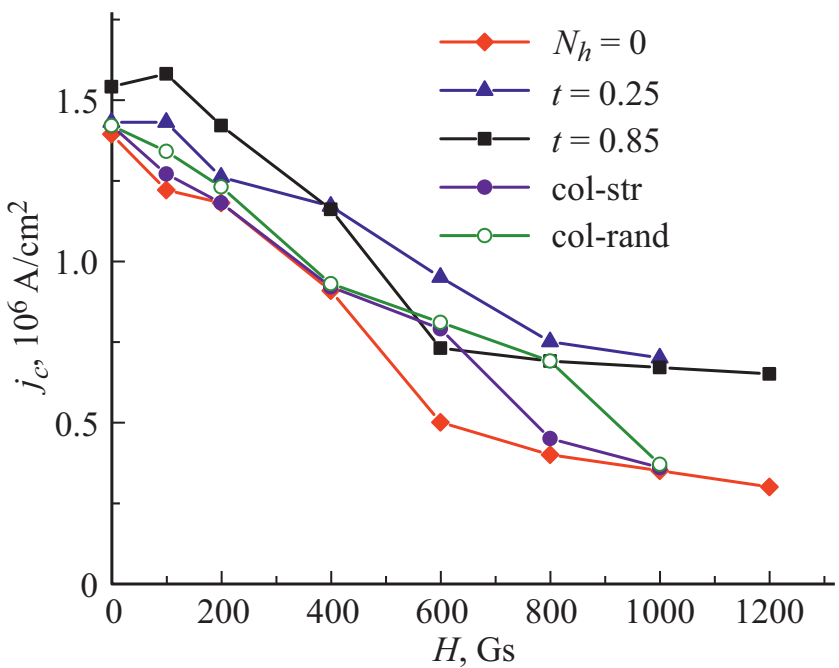

Pис. 4. Сравнение зависимостей критического тока от магнитного поля для необлученного образца $\left(N_{h}=0\right)$, облученного ионами с двумя разными значениями относительной глубины конического дефекта, с радиационными дефектами типа А (col-str) и типа B (col-rand).

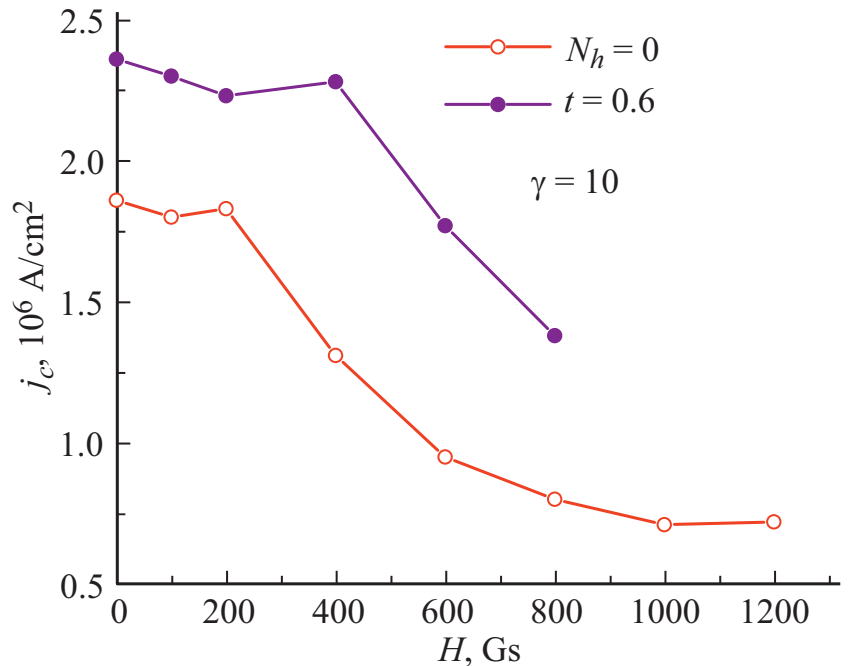

Рис. 5. Зависимость критического тока от магнитного поля для конических дефектов, слабоанизотропный случай. $N_{h}=0-$ необлученный сверхпроводник, $t=0.6-20$ конических дефектов с относительной глубиной $h / c=0.6$.
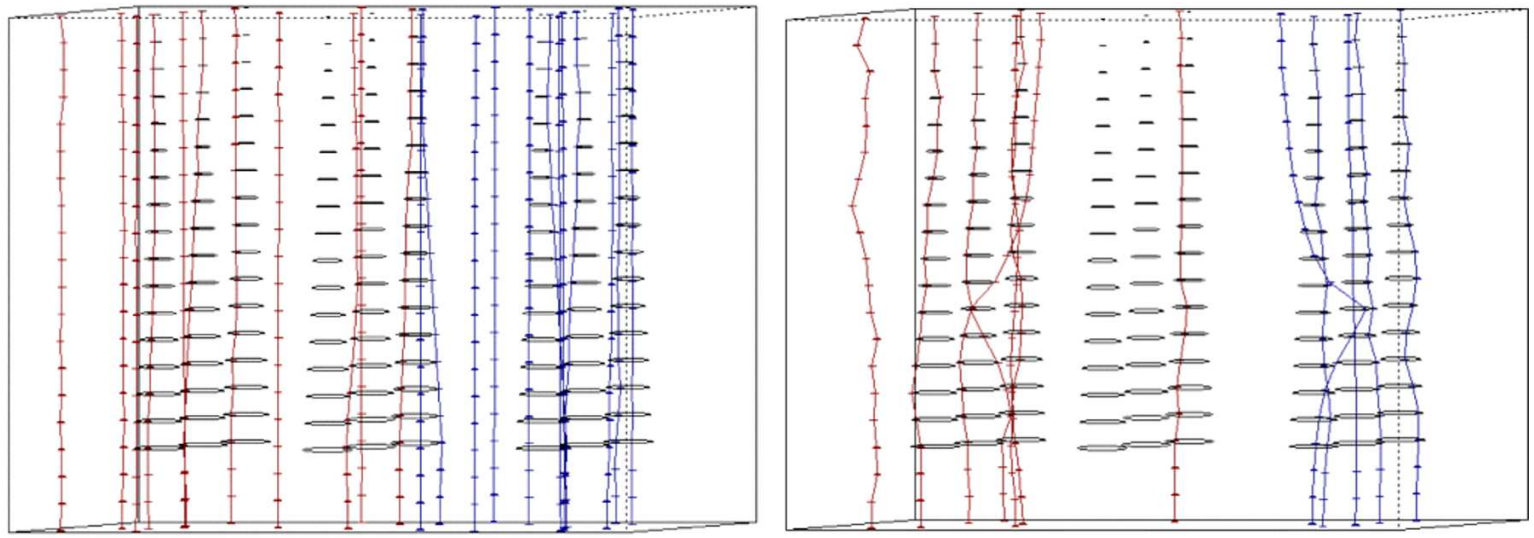

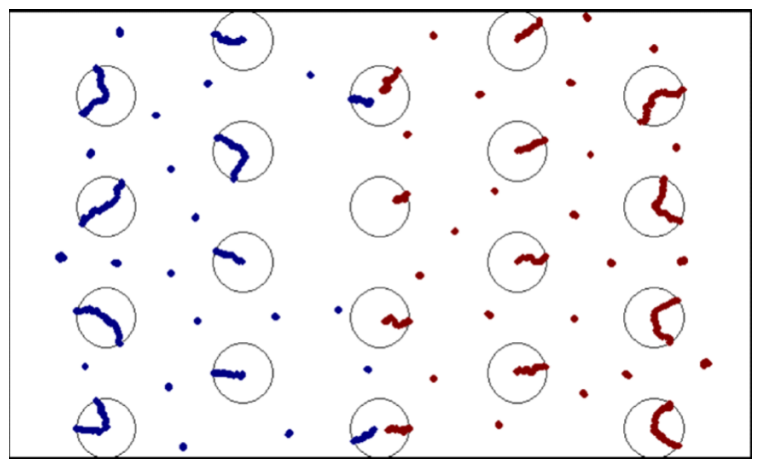

YBCO

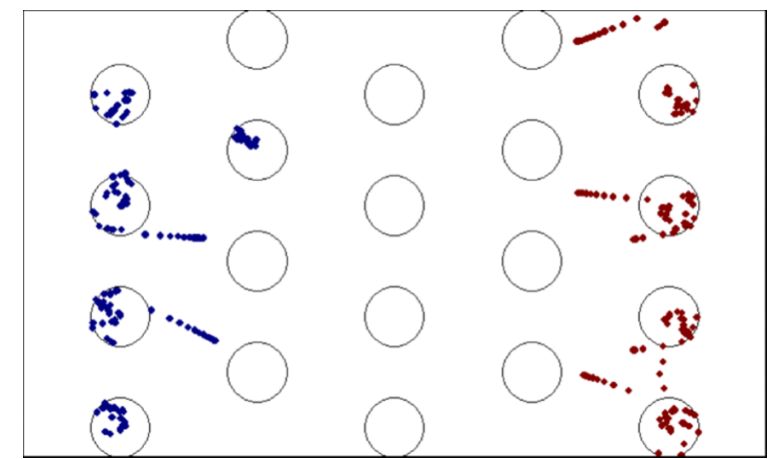

BSCCO

Рис. 6. Вихревые конфигурации, возникающие при перемагничивании током, $t=0.85$, собственные дефекты отсутствуют. На верхних рисунках показаны трехмерные вихревые нити, взаимодействующие с коническими дефектами. Круги растущего радиуса означают области с подавленной сверхпроводимостью в каждом ВТСП-слое. На нижнем рисунке (вид сверху на образец) кругами обозначены нижние, самые широкие части дефектов. Красные и синие закрашенные круги - панкейки. Чтобы не загромождать рисунок, на трехмерных конфигурациях показано меньшее число вихрей и дефектов, чем на нижних рисунках. 
Таким образом, при эквивалентной концентрации конические дефекты наиболее эффективны для повышения критического тока, как сильноанизотропном, так и в слабоанизотропном случае. Для качественного объяснения этого результата проанализируем далее закрепление вихрей на конических дефектах. Для этого сделаем мгновенные снимки вихревой структуры при поле тока, немного превышающим первое критическое, когда в образце не слишком много вихрей. Рассмотрим случаи низкой (YВСО) и высокой (BSCCO) анизотропии (рис. 6). При малой анизотропии вихревая нить жесткая, так что такие вихри либо не закрепляются на дефектах вовсе, либо способ закрепления соответствует минимальному их изгибу (и обеспечивает более сильный пиннинг и критический ток). Таким образом, жесткие вихри закрепляются на дефектах вдоль образующей конуса, что дополнительно иллюстрирует вид сверху на плоскость ab. При высокой анизотропии наблюдаются множественные изгибы вихрей, частичное закрепление на дефекте, закрепление вихря одновременно на нескольких дефектах, что приводит к снижению пиннинга.

\section{4. Заключение}

Численно исследовано взаимодействие вихревой решетки в высокотемпературном сверхпроводнике с радиационными дефектами, выполнены расчеты критического тока и вихревых конфигураций. Детально исследован случай дефектов, возникающих при облучении ионами относительно низкой энергии (несколько десятков $\mathrm{Mev}$, конические дефекты). Получены следующие результаты.

1. Повышение критического тока для конических дефектов составляет примерно 30\%. При эквивалентном флюенсе облучения для конических дефектов наблюдается наибольшее увеличение критического тока по сравнению с колончатыми и случайными.

2. При слабом собственном пиннинге для глубокого дефекта наблюдается при низких полях слабый рост критического тока с ростом поля.

3. Облучение ионами низкой энергии при эквивалентном флюенсе повышает критический ток на $10 \%$ сильнее для сверхпроводника с низкой анизотропией.

4. Конические дефекты большей глубины при низких полях более эффективны для усиления пиннинга.

\section{Финансирование работы}

Исследование выполнено при финансовой поддержке РФФИ в рамках научного проекта № 20-0800811 (И.А. Руднев), а также при финансовой поддержке РФФИ и Госкорпорации „Росатом“ в рамках научного проекта № 20-21-00085 (В.А. Кашурников, А.Н. Мороз).

\section{Конфликт интересов}

Авторы заявляют, что у них нет конфликта интересов.

\section{Список литературы}

[1] J.S. Umezawa, G.W. Crabtree, J.Z. Liu, H.W. Weber, W.K. Kwok, L.H. Nunez, T.J. Moran, C.H. Sowers, H. Claus. Phys. Rev. B 36, 7151 (1987).

[2] B.M. Vlcek, H.K. Viswananthan, M.C. Frischherz, S. Fleshler, K. Vandervoort, J. Downey, U. Welp, M.A. Kirk, G.W. Crabtree. Phys. Rev. B 40, 67 (1993).

[3] J. Giapintzakis, W.C. Lee, J.P. Rice, D.M. Ginsberg, I.M. Robertson, M.A. Kirk, R. Wheeler. Phys. Rev. B 45, 10677 (1992).

[4] M.K. Hasan, J. Shobaki, I.A. Al-Omari, B.A. Albiss, M.A. AlAkhras, K.A. Azez, A.K. El-Qisari, J.S. Kouvel. Supercond. Sci. Technol. 12, 606 (1999).

[5] L. Civale, A.D. Marwick, T.K. Worthington, M.A. Kirk, J.R. Thompson, L. Krusin-Elbaum, Y.R. Sun, J.R. Clem, F. Holtzber. Phys. Rev. Lett. 67, 648 (1991).

[6] M.P. Smylie, M. Leroux, M. Mishra, L. Fang, M. Taddei, O. Chmaissem, W.K. Kwok. Phys. Rev. B, 93, 11, 115119 (2016).

[7] P. Biersack, L.G. Haggmark. Nucl. Instrum. Meth. Phys. Res. B 74, 257 (1980). WWW.srim.org

[8] А.И. Подливаев, И.А. Руднев. ФТТ 63, 6, 712 (2021).

[9] M.C.H.W.W.M. Eisterer, R. Fuger, M. Chudy, F. Hengstberger, H.W. Weber. Supercond.Sci. Technol. 23, 1, 014009 (2009).

[10] M. Chudy, R. Fuger, M. Eisterer, H.W. Weber. IEEE Transact. Appl. Supercond. 21, 3, 3162 (2011).

[11] R. Prokopec, D.X. Fischer, H.W. Weber, M. Eisterer. Supercond. Sci. Technol. 28, 1, 014005 (2014).

[12] M. Jirsa, M. Rameš, I. Duuran, T. Melíš ek, P. Kováč, L. Viererbl. Supercond. Sci. Technol. 30, 4, 045010 (2017).

[13] K.J. Leonard, F.A. List III, T. Aytug, A.A. Gapud, J.W. Geringer. Nucl. Mater. Energy, 9, 251 (2016).

[14] D.X. Fischer, R. Prokopec, J. Emhofer, M. Eisterer. Supercond. Sci. Technol. 31, 4, 044006 (2018).

[15] J. Emhofer, M. Eisterer, H.W. Weber. Supercond. Sci. Technol. 26, 3, 035009 (2013).

[16] I.A. Rudnev, D.S. Odintsov, V.A. Kashurnikov. Phys. Lett. A, 372, 21, 3934 (2008).

[17] J. Trastoy, V. Rouco, C. Ulysse, R. Bernard, G. Faini, J. Lesueur, J. Briatico, J.E. Villegas. Physica C 506, 15, 195 (2014). http://dx.doi.org/10.1016/j.physc.2014.06.016

[18] N. Haberkorn, S. Suarez, S.L. Bud'ko, P.C. Canfield. Solid State Commun. 318, 113963 (2020).

[19] M. Wacenovsky, R. Miletich, H.W. Weber, M. Murakami. Cryogenics 33, 1, 706 (1993).

[20] B. Shao, A. Liu, H. Ren, Q. He, L. Xiao, T. Takeyama. Mater. Res. Bull. 27, 1, 15 (1992).

[21] K. Takase, K. Demachi, K. Miya. Cryogenics 39, 5, 435 (1999).

[22] K.L. Mletschnig, W. Lang. Microelectron. Eng. 215, 110982 (2019).

[23] V. Sandu, A.M. Ionescu, I. Ivan, L. Craciun, G. Aldica. Physica C 578, 1353734 (2020).

[24] K.R. Mavani, D.S. Rana, S. Rayaprol, R.N. Parmar, D.G. Kuberkar, R. Kumar, R. Nagarajan. Solid State Commun. 142, 8,462 (2007).

[25] G. Mikhailova, L. Antonova, A. Troitskii, A. Didyk, V. Malginov, T. Demikhov, E. Suvorova. Physica Status Solidi C, 10, 4, 677 (2013). 
[26] A.V. Troitskii, L.K. Antonova, T.E. Demikhov, V.A. Skuratov, V.K. Semina, G.N. Mikhailova. Physica C 572, 1353631 (2020).

[27] N. Haberkorn, S. Suárez, P.D. Pérez, H. Troiani, P. Granell, F. Golmar, S.H. Moon. Physica C 542, 6, 11 (2017).

[28] M. Leroux, K.J. Kihlstrom, S. Holleis, M.W. Rupich, S. Sathyamurthy, S. Fleshler, W.K. Kwok. Appl. Phys. Lett. 107, 19, 192601 (2015).

[29] S. Eley, M. Leroux, M.W. Rupich, D.J. Miller, H. Sheng, P.M. Niraula, L. Civale. Supercond. Sci. Technol. 30, 1, 015010 (2016).

[30] N. Haberkorn, S. Suárez, J.H. Lee, S.H. Moon, H. Lee. Solid State Commun. 289, 51 (2019).

[31] I.A. Sadovskyy, Y. Jia, M. Leroux, J. Kwon, H. Hu, L. Fang, W.K. Kwok. Adv. Mater. 28, 23, 4593 (2016).

[32] W.E. Lawrence, S. Doniach. In: Proceedings of LT 12, Kyoto, 1970 / Ed. E. Kanda. Keigaku, Tokyo (1971). 361 p.

[33] V.A. Kashurnikov, A.N. Maksimova, I.A. Rudnev, D.S. Odintsov. IEEE Transact. Appl. Supercond. 26, 3, 8200404, 1 (2016)

[34] В.А. Кашурников, А.Н. Максимова, И.А. Руднев. ФТТ 56, $861(2014)$

[35] A.N. Moroz, A.N. Maksimova, V.A. Kashurnikov, I.A. Rudnev. IEEE Trans. Appl. Supercond. 28, 8000705 (2018)

[36] Sandeep Tyagi, Yadin Y. Goldschmidt. Phys. Rev. B 70, 024501, 1 (2004)

[37] Yadin Y. Goldschmidt, Sandeep Tyagi. Phys. Rev. B 71, 014503, 1 (2005)

[38] G. Blatter, M.V. Feigel'man, V.B. Geshkenbein, A.I. Larkin, V.M. Vinokur. Rev. Mod. Phys. 66, 4, 1125 (1994)

[39] E.I. Suvorova, P.N. Degtyarenko, I.A. Karateev, A.V. Ovcharov, A.L. Vasiliev, V.A. Skuratov, Ph.A. Buffat. J. Appl. Phys. 126, 145106 (2019)

Редактор Т.Н. Василевская 